\title{
Mupirocin Resistance in Staphylococcus aureus isolated from the Anterior Nares of Health Care Workers, in a Tertiary Care Hospital
}

\author{
Ragini Ananth Kashid ${ }^{1 *}$ and Kausalya Raghuraman ${ }^{2}$ \\ ${ }^{1}$ Department of Microbiology, BGS Global Institute of Medical Sciences, No.67, BGS Health \\ and Education City, Uttarahalli Main Road, Kengeri Bangalore - 560060, India \\ ${ }^{2}$ Department of Microbiology, Raja Rajeswari Medical College and Hospital, Kambipura, \\ Mysore Road, Bangalore-560074, India \\ *Corresponding author
}

\begin{tabular}{|l|}
\hline Ke y w o r d s \\
$\begin{array}{l}\text { Anterior nares, Health } \\
\text { care workers, High } \\
\text { level mupirocin } \\
\text { resistance, Low level } \\
\text { mupirocin resistance, } \\
\text { Methicillin resistant, } \\
\text { Staphylococcus aureus }\end{array}$ \\
\hline Article Info \\
\hline $\begin{array}{l}\text { Accepted: } \\
\text { 04 January } 2018 \\
\text { Available Online: } \\
\text { 10 February } 2018\end{array}$ \\
\hline
\end{tabular}

A B S T R A C T
Nasal carriage of methicillin resistant Staphylococcus aureus (MRSA) is a key factor in the epidemiology and causation of infection. Health care workers, who are nasal carriers of MRSA, act as reservoirs of infection. This is an important risk factor in the development of nosocomial infections. Mupirocin is used to decolonise MRSA carriers. But with the widespread use of mupirocin, resistance to mupirocin has set in. This poses a challenge to the use of mupirocin against MRSA. This study was carried out to know the occurrence of high and low level mupirocin resistance in Staphylococcus aureus. Staphylococcus aureus isolated from the anterior nares of health care workers (HCWs) were identified using standard protocol. Methicillin resistance was identified using cefoxitin disc $(30 \mu \mathrm{g})$. High level and low level mupirocin resistance was identified using $5 \mu \mathrm{g}$ and $200 \mu \mathrm{g}$ mupirocin discs. The tests were done by Kirby Bauer disc diffusion method as per CLSI guidelines. SPSS Version 20 software was used to give percentage analysis of the data. Chi-square test was used for statistical analysis ( $\mathrm{p}$ value $<=0.05$ is taken as statistically significant). Of the 300 nasal swabs taken from HCWs, 28 Staphylococcus aureus were isolated, of which 24 isolates were MSSA and 4 isolates were MRSA. One MSSA (1/24, 4.1\%) isolate showed high level mupirocin resistance. Only 1 MRSA isolate $(1 / 4,25 \%)$ had high level resistance to mupirocin. No low level resistance to mupirocin was detected in the MSSA and MRSA isolates. Presence of mupirocin resistance in Staphylococcus aureus isolates is a cause of grave concern. The hospital infection control activities and surveillance have to be stepped up, to identify and control mupirocin resistance.

\section{Introduction}

Staphylococcus aureus is a well-known nosocomial pathogen with high mortality and morbidity. It increases the treatment cost and prolongs the hospital stay of the patient. Nasal carriage of Staphylococcus among health care workers is one of the means of transmission of this deadly organism. Colonized individuals (patient or health care worker) serve as a potential reservoir of infection, even if they are asymptomatic (Agarwal et al., 2015). Mupirocin is used for the decolonization of Staphylococcus from anterior nares of health 
care workers (Kaur, 2014). Mupirocin (Pseudomonic acid A) is produced by Pseudomonas fluorescence. It is used for the treatment of skin and soft tissue infection caused by Staphylococcus spp. and Streptococcus spp. It is also used to decolonise nasal carriage of methicillin resistant Staphylococcus aureus (MRSA). Mupirocin, an analogue of isoleucine, acts by inhibiting protein synthesis, as it competitively binds to isoleucine t- RNA synthetase (IRS) (Sanju et al., 2015; Wattal, 2014).

United Kingdom was the first country to introduce mupirocin in 1985 (Sanju et al., 2015). Within a short span of 2 years, the emergence of drug resistance was reported (Wattal, 2014). The widespread use of mupirocin as a topical decolonizing agent for MRSA in health care facilities has led to emergence of mupirocin resistance (Kaur, 2014). Resistance to mupirocin can be high level resistance and low level resistance, based on the zone diameter or minimum inhibitory concentration (MIC). Mupirocin sensitive isolates have a zone diameter $\geq 14 \mathrm{~mm}$ for both $5 \mu \mathrm{g}$ and $200 \mu \mathrm{g}$ discs or have a MIC $\leq 4 \mu \mathrm{g} / \mathrm{ml}$. Low level mupirocin resistant $(\mathrm{MuL})$ isolates show a zone diameter $<14 \mathrm{~mm}$ in the $5 \mu \mathrm{g}$ disc, but more than or equal to $14 \mathrm{~mm}$ in the $200 \mu \mathrm{g}$ disc or have a MIC of $8-256 \mu \mathrm{g} / \mathrm{ml}$. High level mupirocin resistant $(\mathrm{MuH})$ isolates that show zone diameter $<14 \mathrm{~mm}$ in both the $5 \mu \mathrm{g}$ disc and $200 \mu \mathrm{g}$ disc or have a MIC $\geq 512$ $\mu \mathrm{g} / \mathrm{ml}$ (Kaur, 2014, Sanju et al., 2015, Wattal, 2014). High level mupirocin resistance is by mup A gene, which is plasmid mediated and codes for a novel IRS. Low level mupirocin resistance is associated with a chromosomal point mutation associated with a change in the native IRS. Novel gene, тир B has been identified, also being responsible for high level resistance (Wattal, 2014)

The plasmid which harbours the Mup A gene is known to carry resistance to other antibiotics like macrolide, gentamicin, tetracycline and trimethoprim, thus increasing the drug resistance (Wattal, 2014). Detection of low level mupirocin resistance is important as nasal isolates can still be controlled with mupirocin therapy as the ointment has a higher mupirocin concentration $(20,000$ $\mu \mathrm{g} / \mathrm{ml}$ ) (Oliveria et al., 2007).

The clinical relevance of detecting mupirocin resistance is that in high level mupirocin resistance, patients on mupirocin therapy are unable to clear the organism (Oliveria et al., 2007; Malaviolle et al., 2008; Simor et al., 2007). Few studies have suggested that low level mupirocin resistance has been detected, can predict treatment failure (Malaviolle et al., 2008; Simor et al., 2007). Resistance to mupirocin ranges from 8.3-10\% (Krishnan et al., 2002).

It has been observed that the prevalence of colonization by MRSA in HCWs varies with different locations and with the institution (Kaur, 2014). Therefore, there is a need to detect both high level and low level mupirocin resistance among health care workers and to treat them with other alternate decolonizing agent. Hence, the present study was undertaken to know the occurrence of mupirocin resistance in those health care workers, whose anterior nares were colonized with Staphylococcus aureus. This data will help our hospital infection control team to devise more effective strategies to control MRSA and to keep alternate therapeutic options ready, to keep mupirocin resistance in check.

\section{Materials and Methods}

A prospective study was conducted for duration of 6 months among health care workers, in a tertiary care hospital, south India. All the health care workers who had given an informed written consent were 
enrolled in the study. Health care workers with recent history of nasal surgery, fever, upper respiratory tract infection and on any topical medication were excluded from the study. The study was cleared by the institutional ethical committee.

Using a prepositioned swab, samples were collected from the anterior nares of the health care workers. The samples were inoculated on MacConkey agar, blood agar and mannitol salt agar. Staphylococcus aureus was identified using the standard microbiological technique.

Methicillin resistance was detected as per CLSI guidelines using cefoxitin disc diffusion method. A $0.5 \mathrm{McFarland}$ standard suspension of the isolate was prepared. A lawn culture on Mueller Hinton agar was prepared with the standard inoculum, and a cefoxitin disc $(30 \mu \mathrm{g}$, Himedia, Mumbai, India) was placed. After overnight incubation, a zone diameter of $<=21 \mathrm{~mm}$ was considered resistant and an inhibition zone $=>22 \mathrm{~mm}$ was considered as methicillin sensitive.

Resistance to mupirocin was tested using two different strengths of mupirocin disc: mupirocin $5 \mu \mathrm{g}$ (Himedia, Mumbai, India) and mupirocin $200 \mu \mathrm{g}$ (Oxoid, Basingstroke, UK). A $0.5 \mathrm{McFarland}$ standard suspension of the isolate was prepared, was lawn cultured on Mueller Hinton agar. The mupirocin $5 \mu \mathrm{g}$ and mupirocin $200 \mu \mathrm{g}$ discs were placed on the plate. After overnight incubation the zone of inhibition was measured. An inhibition zone of $<=13 \mathrm{~mm}$ is considered as mupirocin resistance and an inhibition zone of $=>14 \mathrm{~mm}$ is considered as sensitive (Oliveria et al., 2007).

\section{Statistical analysis}

The results were recorded and analysed statistically using Microsoft excel sheet 2009. Percentage description of data was given using
SPSS Version 20 software. Chi-square test was used for statistical analysis. A p value <= 0.05 is taken as statistically significant.

\section{Results and Discussion}

A total of 300 nasal swabs were collected from health care workers and were processed as per standard microbiological procedures. Of the 300 nasal swabs taken, we isolated 28 Staphylococcus aureus (9.33\%). Among the 28 Staphylococcus aureus isolates, only 4 strains were found to be Methicillin resistant Staphylococcus aureus (MRSA) and the remaining 24 strains was Methicillin sensitive Staphylococcus aureus (MSSA). The overall positivity of MRSA was $1.33 \%(4 / 300)$ and the positivity of MSSA was $8 \%$ (24/300) (Table 1).

Among the 24 strains of MSSA, high level mupirocin resistance was found in one isolate. The overall positivity of mupirocin resistance in MSSA was $4.1 \%$. Out of the 4 MRSA isolated only 1 isolate had high level resistance to mupirocin. The overall positivity to mupirocin resistance among MRSA was 25 $\%$ (Table 2). No low level resistance was detected in the MSSA and MRSA isolates (Table 3).

Nasal carriers of Staphylococcus aureus are three times more prone to infection than noncarriers. It is observed that almost $30 \%$ of the world population is persistently or occasionally colonized with Staphylococcus aureus strains in the anterior nares (Trouillet Assant et al., 2015). Various decolonizing agents used against Staphylococcus aureus include chlorhexidine, mupirocin, rifampin, povidone iodine and doxycycline (Vedavati et al., 2014). Although some have been used for nasal carriage, they show good response only for a short period of time and recolonization is seen again (Schmitz et al., 1998). Ability to eliminate $97 \%$ Staphylococcus aureus in the 
anterior nares in health care workers, within 24 hours, makes mupirocin the best drug for decolonization (Vedavati et al., 2014). Mupirocin can be used for decolonization of both MSSA and MRSA isolates (Hetem, 2013). Lately it has been used for pre surgical decolonization and there is a 58\% reduction in the rate of post - surgical infection. It is also being used in dialysis patients (haemodialysis and peritoneal dialysis), to prevent infections in these patients (Hetem, 2013, Wattal, 2014).

Resistance to mupirocin is reported to be $5 \%$ in Korea, 6\% in India, 6.6\% in China, $11.3 \%$ in Spain, $13.2 \%$ in United States of America, $26.1 \%$ in Trinidad Tobago and $45 \%$ in Turkey (Rajkumari et al., 2014). Although the wide spread use of mupirocin has led to resistance, the other causes of mupirocin resistance are: concentration of the drug in deeper layers of skin is less than in nose, irregular and ineffective application (mupirocin need to be massaged back on the nose for at least $1 \mathrm{~min}$ ), reinfection due to a different strain with resistance and rarely by aerosol spread especially in dermatology and burns ward (Cookson, 1998). Even when mupirocin is applied in clinically effective concentration in the nose, it will lead to the presence of low level of antibiotic in the pharynx, which could induce or select resistant strains (Oommen et al., 2010). Few others believe that blanket treatment on all health care workers or patients with MRSA carriage has resulted in the resistance (Orett, 2008).

It is well proven that screening with $5 \mu \mathrm{g}$ disc of mupirocin cannot differentiate between MuL and MuH strains. Therefore, we need to use both $5 \mu \mathrm{g}$ and $200 \mu \mathrm{g}$ disc for the same (Sanju et al., 2015).

Table.1 Overall sensitivity to methicillin in the isolates of Staphylococcus aureus

\begin{tabular}{|c|c|c|c|}
\hline $\begin{array}{l}\text { Organism isolated } \\
(\mathbf{n = 3 0 0 )}\end{array}$ & $\begin{array}{c}\text { Methicillin } \\
\text { sensitive }(\%)\end{array}$ & $\begin{array}{c}\text { Methicillin resistant } \\
(\%)\end{array}$ & \begin{tabular}{c} 
Total \\
\hline Staphylococcus aureus
\end{tabular} \\
\hline $24(8)$ & $04(1.33)$ & 28 \\
\hline
\end{tabular}

Table.2 Percentage of mupirocin resistance in MRSA versus MSSA with the p value

\begin{tabular}{|c|c|c|c|c|c|}
\hline \multirow{2}{*}{ Mupirocin } & \multicolumn{3}{|c|}{ MIRA } & \multicolumn{3}{c|}{ MSSA } & p value \\
\hline & Number & Percentage (\%) & Number & Percentage (\%) & \\
\hline Resistant & 1 & 25 & 1 & 4.1 & 0.1342 \\
\hline Sensitive & 3 & 75 & 23 & 95.9 & \\
\hline Total & 4 & 100 & 24 & 100 & \\
\hline
\end{tabular}

Table.3 Distribution of MuL and MuH resistant isolates of MSSA and MRSA

\begin{tabular}{|c|c|c|c|}
\hline $\begin{array}{l}\text { Methicillin } \\
\text { sensitivity }\end{array}$ & $\begin{array}{l}\text { Mupirocin } \\
\text { resistant (\%) }\end{array}$ & $\begin{array}{l}\text { Low level mupirocin } \\
\text { resistant (MuL) }\end{array}$ & $\begin{array}{l}\text { High level mupirocin } \\
\text { resistant (MuH) }\end{array}$ \\
\hline MRSA (04) & $1(25)$ & 0 & 1 \\
\hline MSSA (24) & $1(4.1)$ & 0 & 1 \\
\hline
\end{tabular}


Table.4 Distribution of mupirocin resistance $(\mathrm{MuL}$ and $\mathrm{MuH})$ in various studies

\begin{tabular}{|c|c|c|c|c|}
\hline \multirow{2}{*}{ Studies } & \multicolumn{2}{|l|}{ MSSA } & \multicolumn{2}{|l|}{ MRSA } \\
\hline & $\begin{array}{l}\text { Low } \\
\text { (MuL) }\end{array}$ & $\begin{array}{l}\text { High } \\
\text { (MuH) }\end{array}$ & $\begin{array}{l}\text { Low } \\
\text { (MuL) }\end{array}$ & $\begin{array}{l}\text { High } \\
\text { (MuH) }\end{array}$ \\
\hline Present study & 0 & $1(4.1 \%)$ & 0 & $1(25 \%)$ \\
\hline Schmitz FJ et al., (1998) & $9(1.8 \%)$ & $6(1.2 \%)$ & $7(3.6 \%)$ & $5(2.6 \%)$ \\
\hline & $1(1.5 \%)$ & $1(1$. & 0 & $1(2.2 \%)$ \\
\hline Kaur DC, ( & 0 & 0 & $1(1.43 \%)$ & $1(1.43 \%)$ \\
\hline Sanju AJ et al.,(2015) & 0 & 0 & $4(14.2 \%)$ & $7(25 \%)$ \\
\hline Oommen SK et al., (2010) & 0 & 0 & 0 & $1(2.08 \%)$ \\
\hline
\end{tabular}

In our study MRSA was $1.33 \%$ which is similar to the study done by Hetem et al., (2013) who showed 3\% resistance rate. High level mupirocin resistance was seen both in MSSA and MRSA in our study. Among MSSA, $4.1 \%(1 / 24)$ of the isolates showed high level resistance. This is comparable with the study done by Schmitz FJ et al., (1998) and Jayakumar et al., (2013).

Among the 4 MRSA isolates in our study, one isolate $(1 / 4,25 \%)$ had produced high level mupirocin resistance. This is comparable with the study conducted by Sanju et al., (2015).Among the 125 MRSA studied by Malaviolle et al., (2008), high level mupirocin resistance was found in $25.6 \%$ and low level mupirocin resistance in $31.2 \%$. Agarwal et al., (2015) studied 28 MRSA isolates and found resistance in 4 isolates. Among the 4 isolates, $75 \%$ resistance was high level mupirocin resistance (Agarwal et al., 2015) (Table 4). The risk of mupirocin resistance is more with MRSA compared to their counterpart MSSA (Kaur, 2014). Resistance may be transferred between the Staphylococcus species from sensitive to resistant strain. Hence, testing for MSSA becomes important. Conjugative plasmids are known to transmit resistance between Staphylococcus aureus and Coagulase negative Staphylococcus (Jayakumar et al., 2013). Hence, when mupirocin resistance is encountered, alternative treatment regimen is sought. Alternative treatment includes chlorhexidine, fusidic acid, neomycin cream and reptapumilin (Wattal, 2014; Oommen et al., 2010). These alternative treatments are used when colonization persists even after 2 courses of mupirocin treatment or when the isolate is mupirocin resistant (Kaur, 2014).

Therefore, we conclude that screening for both high and low level mupirocin resistance is warranted and formulation of appropriate decontamination measures, in case of resistance is required. This will help in prevention and containment of these resistant strains in the hospital setup, leading to better infection control measures and better patient outcomes.

\section{Acknowledgement}

We would like to acknowledge our Chairman Shri A. C. Shanmugam who has always encouraged research activities in our college. We are grateful to the staff of the Department of Microbiology for their constant support.

\section{References}

Agarwal L, Singh AK, Sengupta C, Agarwal A. Nasal carriage of methicillin and mupirocin resistance Staphylococcus aureus among health care workers in a tertiary care hospital. J Res Pharm Pract 2015; 4:182-6.

Cookson BD. The emergence of mupirocin 
resistance: a challenge to infection control and antibiotic prescribing practice. $\mathbf{J}$ of Antimicrobial Chemo 1998; 41:11-18.

Hetem DJ, Bonten MJM. Clinical relevance of mupirocin resistance in Staphylococcus aureus. J of Hosp Infect 2013; 85:249-256.

Jayakumar S, Meerabhai M, Shameem Banu AS, Mathur R, Kalyani M, Binesh LY. Prevalence of high and low level mupirocin resistance among Staphylococcus isolates from skin infection in a tertiary care hospital. J Clin Diag Res 2013; 7(2):238-242.

Kaur DC, Narayan PA. Mupirocin resistance in nasal carriage of Staphylococcus aureus among health care workers of a tertiary care rural hospital. Indian J Crit Care Med 2014; 18(11):716-721.

Krishnan PU, Miles K, Shetty N. Detection of methicillin and mupirocin resistance in Staphylococcus aureus isolates using conventional and molecular methods a descriptive study from a burns unit with high prevalence of MRSA. J Clin Pathol 2002; 55:745-748

Malaviolle X, Nonhoff C, Denis O, Rottiers S, Struelens MJ. Evaluation of disc diffusion methods and Vitek 2 automated system for testing sensitivity to mupirocin in Staphylococcus aureus. J of Antimicrobiol Chemo 2008; 62:1018-23.

Oliveria NEM, Cardozo APCM, Marques EA, Santos KRN, Giambiagi-de Marval M. Interpretive criteria to differentiate low and high level mupirocin resistance in Staphylococcus aureus. J Med Microbiol 2007; 56:937-939.

Oommen SK, Appalaraju B, Jinsha K. Mupirocin resistance in clinical isolates of Staphylococcus in a tertiary care centre in South India. Indian J Med Microbiol 2010; 28(4); 372-5.
Orett FA. The emergence of mupirocin resistance among clinical isolates of MRSA in Trinidad: a first report. Jpn J Infect Dis 2008; 61:107-110.

Rajkumari N, Mathur P, Bhardwaj N, Gupta G, Dahiya $\mathrm{R}$ et al., Resistance pattern of mupirocin in MRSA in trauma patients and comparison between disc diffusion and $\mathrm{E}$ test for better detection of resistance in low resource countries. J lab Physicians 2014; 6(2):91-95.

Sanju AJ, Kopula SS, Palraj KK. Screening for mupirocin resistance in Staphylococcus. J Clin Diag Res 2015; 9(10): DC09-DC 10.

Schmitz FJ, Lindenlaul E, Hofmann B, Fluit AC, Verhoel J, Heinz HP et al., The prevalence of low and high level mupirocin resistance in Staphylococci from 19 European hospitals. J Antimicrob Chem 1998; 42: 489-495.

Simor AE, Stuart TL, Louie L, Watt C, Agostini MO. Mupirocin resistance, MRSA strains in Canadian Hospital. Antimicrob Agents Chemo 2007; 51:3880-3886.

Trouillet-Assant S, Flammier S, Sapin A, Dupiex $\mathrm{C}$, Dumistrescence $\mathrm{O}$ et al., Mupirocin resistance in isolates of Staphylococcus species from nasal swabs in a tertiary care hospital in France. J Clin Microb 2015; 53(8):2713-2715.

Vedavati BI, Kumari AB, Venkatesha D. In vitro activity of mupirocin on Staphylococcus nasal carriers among health care providers of post-operative surgical ward in a tertiary care hospital. Int $\mathbf{J}$ of Allied Med Sci Clin Res 2014; 2(4):282-286.

Wattal C, Oberoi JK. Mupirocin resistant Staphylococcus aureus nasal colonization among health care workers. Indian J Crit Care Med 2014; 18(11): 709-710

\section{How to cite this article:}

Ragini Ananth Kashid and Kausalya Raghuraman. 2018. Mupirocin Resistance in Staphylococcus aureus Isolated from the Anterior Nares of Health Care Workers, in a Tertiary Care Hospital. Int.J.Curr.Microbiol.App.Sci. 7(02): 293-298.

doi: https://doi.org/10.20546/ijcmas.2018.702.038 